\title{
LA TECHNICITÉ DE LA MÉDECINE HIPPOCRATIQUE ET SES LIMITES
}

\author{
SYLVA FISCHEROVÁ
}

\begin{abstract}
The Technicity of the Hippocratic Medicine and Its Limits

The study is concerned with the Hippocratic concept of techne which formed a part of the broader debates on techne occurring in $5^{\text {th }}$ century Greece. The concept of techne is anchored in the concept of physis (and it is at the same time in opposition to the concept of tyche); its importance is attested by the fact that - some time ago - H.-G. Gadamer designated the Greek discovery of techne and its application to medicine as a first decisive commitment towards everything that essentially characterizes Western civilization. What is first presented here are the basic features of techne ietrike (as formulated in the treatises of the Corpus Hippocraticum): Medicine is aware of its goal as well as of its methods whose nature it repeatedly reflects on. What belongs to this reflection is an awareness of certain limits on the part of medicine, especially a lack of akribeia that is due to the fact that doctors deal with objects of a different kind than the other arts do, i.e. the unique constitution of each patient. Another particular of the medical techne is the unequal relationship between the specialist-doctor and the object of his art, the patient. This peculiar situation is commented in the treatises of the Corpus Hippocraticum a number of times. The study's conclusion points out the ethical dimension of dietetic prescriptions and of the medical profession as such; it is something that leads - even in spite of all the limits mentioned above - almost to the deification of both the medical art and the medical life.
\end{abstract}

Keywords: techne; Corpus Hippocraticum; Greek thought of the $5^{\text {th }}$ century $\mathrm{BC}$; medicine and its specific character; ethical dimension of medicine

Dans cet article je vais expliquer en premier lieu la notion hippocratique de la tékhnê et ses charactéristiques spécifiques; puis, je vais éclairer les limites de cette tékhnê que les médecins étaient capable de concevoir et réfléchir ; enfin, je vais discuter les aspects éthiques résultant du caractère spécifique de la tékhnê médicale. 


\section{Tékhnê : les traits essentiels}

Dans le terme tékhnê, on peut reconnaître la racine indoeuropéenne teḱ- (qui signifie produire), ou la racine dupliquée te-t'k- (batir ou charpenter; le substantif téktôn signifie charpentier, menuisier etc., bref : un artisan de profession). Cette racine était très prolifique en latin : considérons à preuve le verbe texo (bâtir et tresser), d'où viennent les mots bien connus, texte et texture (Beekes 2010 : II, 1460 et 1476). On peut traduire tékhnê par art, science ou artisanat, mais aussi, généralement, par expérience, remède, ruse ou embûche. Ce terme caractérise la sphère de l'activité et de la responsabilité humaines, par opposition à la sphère de la nature, phúsis (même si cette opposition n'est pas absolue). Une autre opposition à la tékhnê est représentée par túkhê : le domaine de la fortune et du fortuit.

Les termes désignant les tékhnai singulières commençaient à proliférer au cours du cinquième siècle av. J.-C., les plus anciens d'entre eux étant mousikế (la musique ; chez Pindare, Olymp. I, 15) et mantikế (divination; on la trouve chez Aeschyle, Prom. 484). ${ }^{1}$ Ensuite vinrent s'ajouter nautikế (navigation), rhetorikế etc., et un grand nombre de manuels techniques s'est répandu à partir de ce temps-là.

On peut résumer les traits essentiels de la tékhnê de la manière suivante (mon point de départ est ici l'analyse d'Aristote dans Metaph. I, 1, étant précisé que le Stagirite fonde naturellement ses réflexions sur celles de ses prédecesseurs). ${ }^{2}$

1) la tékhnê a son efficacité, dúnamis, clairement déterminée, et son but, érgon; souvent mais pas toujours - elle a aussi son produit concret, mais il existe des tékhnai qui ne créent pas de produit concret (Arist. Magna mor. 1211b28; Arist. Eth. Eud. 1219a12);

2) tékhnê connaît la route vers ce but, ce qui signifie qu'elle n'est pas seulement une expérience ou empirie, de même qu'elle n'est pas l'affaire du hasard. Dans le même temps se pose la question de l'universalité de cette méthode ainsi que de son exactitude, akríbeia ;

3) elle est capable de transmettre la connaissance acquise par cette méthode aux autres c'est a dire, elle est enseignable, on peut l'enseigner ;

4) elle s'intéresse à son raisonnement, autrement dit : elle questionne sa nature, ses conditions et ses propres limites (cette caractéristique se rattache au point 2).

\section{Les traits essentiels de la tékhnê médicale}

En appliquant ces traits essentiels de la tékhnê à la médecine, voici ce que l'on peut avancer :

1a) le but de la médecine est le suivant: «Délivrer complètement les malades de leurs souffrances ou émousser la violence des maladies, et ne pas traiter les malades qui sont vaincus par les maladies » (De arte 3, traduit par Jouanna 1988). ${ }^{3}$ " Loin d'être

\footnotetext{
Sur la notion de la tékhnê avant Platon voir Heinimann (1961).

On peut consulter aussi l'analyse de Nussbaum (1989: 95).

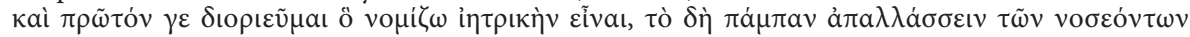

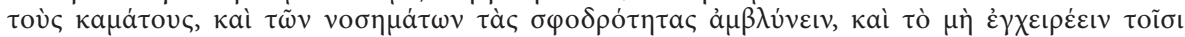

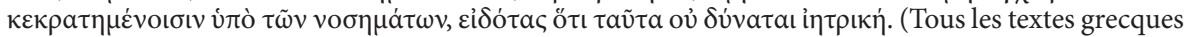
des traités hippocratiques sont pris de Thesaurus Linguae Graecae, c'est à dire de l'édition de Littré 1839-1861.)
} 
une preuve de l'inexistence de l'art comme le prétendent les adversaires, le refus de soigner les cas incurables repose sur la connaissance exacte du domaine qui relève de l'art (cas curables)», commente J. Jouanna ad locum (Jouanna $1988: 249$ ).

Une autre définition célèbre du devoir du médecin va dans le même sens, en disant : « L'art se compose de trois termes: le malade, la maladie et le médecin. Le médecin est le serviteur de l'art; il faut que le malade aide le médecin à combattre la maladie. » (Epid. I, 5) ${ }^{4}$

Cela signifie que le fait de préserver ou d'améliorer la santé n'est pas postulé comme l'érgon de la médecine. Au contraire, le schéma central des médecins grecs présente la maladie comme l'un des angles d'un triangle, les deux angles restants correspondant au malade et au médecin. Chaque angle de ce triangle dispose de la même importance; cela signifie qu'aucun d'eux n'est protégé, ni prépondérant.

2a) La tékhnê médicale est fière d'avoir sa méthode. En fait, les auteurs de plusieurs traités hippocratiques défendent leur tékhnê contre les attaques des adversaires qui maintiennent que le succès du traitement est dû uniquement à la túkhê, pas à la tékhnê médicale (De l'art ; L'ancienne médecine; voir Villard 1996). D’où le caractère clairement apologétique de ces passages.

«La médecine est en possession depuis longtemps de tous ses moyens d'un point de départ et d'une voie qui ont été découverts ; grâce à ces moyens, des découvertes en grand nombre et de belle qualité ont été faites au cours d'une longue période de temps, et les découvertes restantes seront faites pourvu que, joignant à des dons suffisants la connaissance des découvertes acquises, on les prenne pour point de départ de la recherche » (VM 2; traduit par Jouanna 1990). ${ }^{5}$

Cette méthode vise essentiellement à déterminer les causes des processus qui se déroulent dans le corps humain. Il faut trouver ce diá $t i$, "à cause de ", " en raison de " qui rend possible l'analyse de l'état actuel comme la prédiction de l'état prochain, c'està-dire le prognostic. Ces deux notions, le diá ti et le prognostic, sont inséparables l'une de l'autre quoique les médecins grecques (et notamment en ce qui concerne la médecine hippocratique) s'intéressent davantage au prógnôsis qu'au diágnôsis (voir Thivel 1985). En effet, on ne peut lire le terme diágnôsis qu'une seule fois dans le Corpus Hippocraticum: diágnôsin poiéesthai, fixer le diagnostic (VC 10 ; il s'agit d'un traité chirurgical). La cause principale de cette stratégie est enracinée dans la nosologie : on voit et on classifie les maladies davantage comme des faisceaux de symptomes que comme des phenomènes distincts et identifiables de manière univoque. Et voici une information présentant un intérêt tout particulier : on peut également utiliser le terme prodiágnôsis (Vict. 2); ce fait, je l'estime symptomatique per se.

Bref, selon des médecins hippocratiques les choses ne se passent pas autómaton, automatiquement, comme le signale l'auteur du traité De l'art :

4 Toutes les traductions sont par Littré (1839-1861), si ce n'est pas indiqué différemment.

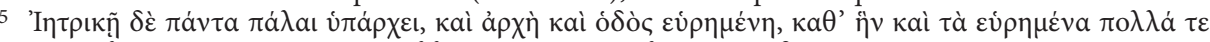

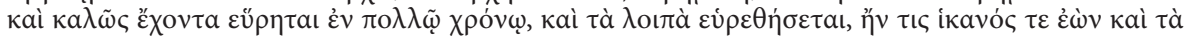

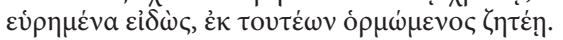




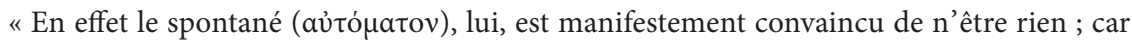
pour tout fait on peut découvrir un pourquoi $(\delta ı \dot{\alpha} \tau \iota)$, et dans la mesure où il y a un pourquoi, le spontané n'a manifestement aucune réalité, si ce n'est en tant que nom » (De arte 6, traduit par Jouanna 1988). ${ }^{6}$

Ce refus de la spontanéité des processus explique la fréquence et l'importance des termes aitía et próphasis, souvent utilisés par les auteurs des traités de Corpus, qui désignent la nécessité, anágkê, des processus dont ils témoignent et qu’ils signalent. On peut rencontrer aussi la distinction sophistiquée entre sunaitía - une cause qui s'associe et accède à la cause primaire - et metaitía, une cause secondaire (Flat. 15).

Ainsi, nous nous sommes positionnés entre les deux concepts fondamentaux de la pensée grecque, phúsis et tékhnê. Phúsis fait figure de notion primordiale et originaire : elle est « l'éducatrice la plus grande de la tékhnê» (Lex 2). Et, dans le traité Du régime, on lit : toutes les tékhnai ont quelque chose en commun avec la nature humaine (Vict. I, 24). C'est pourquoi le principe natura medicatrix revient de manière assez fréquente dans les traités de Corpus Hippocraticum, p. ex. Epid. VI, 5 :

«La nature est le médecin des maladies. [Lit. : Les natures sont les médecins des maladies.] La nature trouve pour elle-même les voies et les moyens, non par intelligence. ... La nature, sans instruction et sans savoir, fait ce qui convient. Larmes, humidité des narines, éternuments, sérumen, salive, expectoration, inspiration, expiration, bâillement, toux, boquet, toutes choses qui ne sont pas toujours de la même nature. $»^{7}$

\section{Tékhnê fonctionne ici comme une imitation de la nature même. ${ }^{8}$}

Pourquoi, peut-on se demander ? C'est parce que chaque tékhnê présuppose phúsis, un ordre des choses qui - même s'il est variable et changeant - renferme les lois de la variation. Phúsis doit, en même temps, être rattachée a l'expérience humaine de sorte que l'expérience humaine permette l'appréhension de la phúsis - et aussi, au niveau empirique, elle permet la réalisation, d'où découlent l'approbation et le succès.

Le devoir du médecin - comme pour chaque technicien - est de discerner la nature de l'instant, c'est-à-dire de l'état donné qui, naturellement, représente toujours " l'histoire de l'état », il résulte de tous les états precédents ; une fois celui-ci appréhendé, le médecin peut fixer la therapie et le prognostic. Le caractère ordonné des choses permet que l'homme puisse le discerner et le reconnaitre ; c'est aussi la raison pour laquelle l'épistemologie est, principalement, ancrée ici. ${ }^{9}$

Bref, comme l'a dit H.-G. Gadamer : la découverte de tékhnê naît de l'esprit de l'histoire ionienne, de l'investigation libre des choses et de l'esprit du lógos; et son application à la médecine constitue le premier engagement décisif pour tout ce qui est essentiel à la

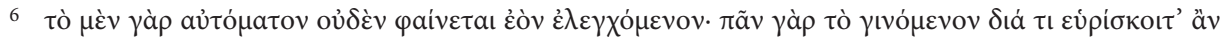

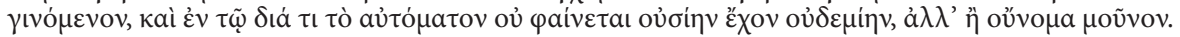

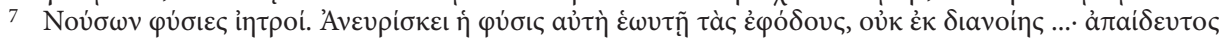

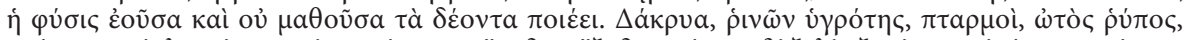

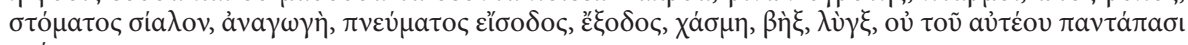
тро́тол.

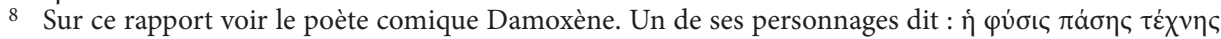

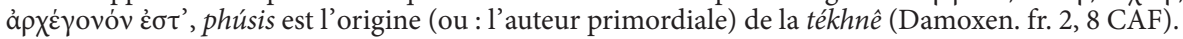

9 Mon point de départ est l'analyse de Kube (1969:44). 
civilisation occidentale (Gadamer 1996 : 31). ${ }^{10}$ Ajoutons encore que dans le concept de la tékhnê on voit aussi opérer la dimension poiétique, c'est à dire pratiquant et « laborant ».

3a) la méthode et le point de départ de l'art médical sont clairs et distincts ; en conséquence, communicables et explicables. Il ne s'agit pas d'initiations secrètes ni d'un traitement par l'extase ou grâce à d'extraordinaires facultés charismatiques du médecin. Si ce n'était pas le cas, non seulement l'enseignement dans les écoles médicales, tel qu'indiqué dans le Serment d'Hippocrate et par d'autres témoignages, mais aussi la totalité du Corpus Hippocraticum seraient incompréhensibles et privés de sens.

4a) L'art médical questionne sa propre nature, ainsi que ses conditions et ses limites ; ce débat dans les traités hippocratiques est riche et nous allons le présenter.

\section{La médecine comme tékhnê « stochastique »}

Une première limite de l'art médical est déterminée par le caractère spécifique de la nature humaine qui implique le manque d'exactitude, akríbeia, de l'art médical. C'est peut-être pour la même raison (et de manière surprenante au premier regard) que les termes akribếs ${ }^{11}$ et akribeía appartiennent aux notions fréquemment mentionnées dans le Corpus Hippocraticum. On les utilise pour désigner l'ordre - la régularité - l'exactitude, p. ex. du cours de la maladie, de sa cure etc. ${ }^{12}$ On peut utiliser akribếs aussi pour classifier les fièvres selon leur durée : une fièvre de trois jours est notée comme tritaĩos akribếs (Epid. ; Aph. Coac.), et nous rencontrons aussi díaita akribếs, le régime régulier (voir Kurz $1970: 68$ ).

Dans la dimension du temps, la mesure est exprimée par la notion de kairós. Kairós peut désigner aussi la mesure per se, mais dans les traités médicales on l'utilise principalement pour exprimer l'occasion, le moment opportun. «Dans le temps est l'occasion ; et dans l'occasion, un temps bref. La guérison se fait dans le temps, parfois aussi dans l'occasion » (Praec. 1). ${ }^{13}$ La médecine elle-même est oligókairos, " elle est d'une mesure fugitive », comme le traduit Brătescu (1983 : 143). Les kairoí sont caractérisés comme polloí et pantoĩoi - ils sont nombreux et de sortes différentes - en correspondance avec les maladies, les lésions et les traitements (Morb. I, 5). Mais on peut nuancer et graduer dans la notion même : il y a les oxées kairoí, les opportunités ou les moments aigus, c'est-à-dire critiques, où il est nécessaire d'intervenir sans hésiter.

10 Sur le caractère « technique » de la médecine contemporaine voir Delkeskamp-Hayes, Gardell Cutter (1993).

11 Le terme akribếs apparaît pour la première fois dans Iliou persis (un des poèmes du cycle homérique, datant probablement du sixième siècle av. J.-C.). Dans le fragment conservé, Poséidon a donné à Machaon - un des fils d'Asclépios - la faculté de retirer les flèches des plaies ; mais, dans la poitrine de Podalire (un autre fils d'Asclépios) il a inséré akribéa pánta - toute la précision. Ça signifie qu'il lui a donné la faculté de discerner l'invisible et de traiter l'incurable, p. ex. la démence d'Aiax (fr. 5 de l'édition d'Allen 1912). Puis que l'adjectif akribếs n'appartient pas à la langue épique, Schiefsky conclut qu'il pourrait être rattaché à la sphère de la médecine (Schiefsky 2005 : 17). A mon avis, la fréquence de cet adjectif et de ses formes graduées chez Hérodote n'indiquent que la provenance ionienne de ce concept important de la pensée grecque.

12 En ce qui concerne le sens originel de l'akríbeia, son etymologie n'est pas connue, mais le terme était utilisé pour qualifier la qualité de produits des tékhnai telles que l'architecture, la charpenterie ou la sculpture, c'est à dire l'harmonie, l'accord harmonieux des détails précisés et la capacité à constituer un ensemble accompli, équilibré et élaboré.

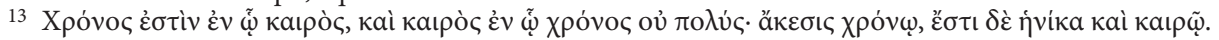


Et - au sommet de cette pyramide - on trouve le superlatif : les oxútatoi kairoí, les moments les plus décisifs, p. ex. quand il s'agit de secourir un patient qui tombe en défaillance, qui ne peut uriner ou aller à la selle, qui étouffe, ou de délivrer une femme qui accouche ou qui se blesse. Là, il ne suffit pas d'intervenir un peu après, car un peu après la plupart a succombé (Morb. I, 5). Mais il y a aussi les maladies pour lesquelles il est impossible de déterminer le khrónos akribếs, parce qu'il dépend de facteurs variés et changeants (Morb. I, 16).

En général, le médecin s'efforce à atteindre la plus haute akríbeia, exactitude et régularité maximales dans son traitement, mais il doit se rattacher à la mesure plus précaire et incertaine que sont le numéro ou le poids, les quantifiants caractérisant une part des tékhnai selon Platon. Dans le Philèbe, Platon subdivise toutes les tékhnai en deux parts (Pl. Phil. 55e-56c) : les premières sont les tékhnai utilisant la mesure, le calcul ou le pesage, bref: les méthodes quantifiantes. Parmi ces tékhnai figurent l'arithmétique, la géométrie, etc. Les secondes sont les tékhnai qui procèdent sur la base de l'estimation approximative ou stochastique (stokhasmós est le terminus technicus ici) et sont issues de l'expérience, de l'exercise et de la pratique (p. ex. mousikế-stratêgikế-kybernêtikế = timonerie).

La médecine appartient elle aussi à cette deuxième partie "stochastique » des tékhnai selon Platon. L'auteur du traité L'ancienne médecine (qui précède le Philèbe) ${ }^{14}$ commente ce caractère spécifique de la tékhnê médicale de manière explicite:

"C'est pourquoi les tâches [scil. de la médecine] sont bien plus diversifiées et requièrent une

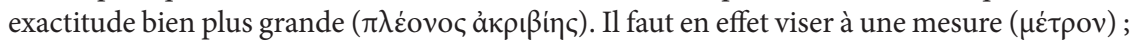
or il n'y a pas de mesure - pas plus du reste qu'un nombre ni qu'un poids -, à quoi on puisse

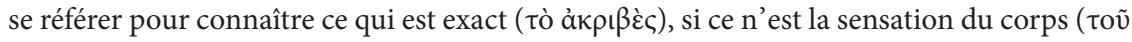

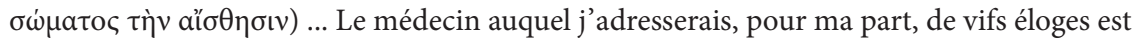

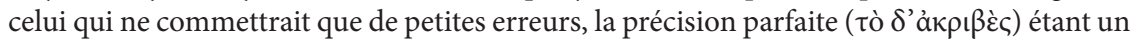
spectacle rare » (VM 9, traduit par Jouanna 1990). ${ }^{15}$

L'auteur du traité discute la même question dans un passage suivant, en utilisant le terme atrekếs (deux fois à la forme superlative) :

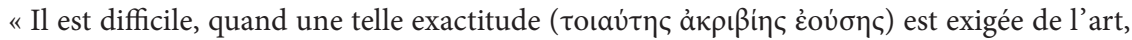

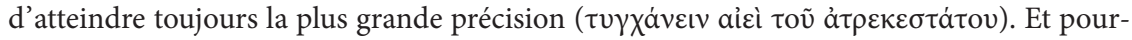
tant, bien des aspects de la médecine, dont il sera question, parviennent à un tel degré

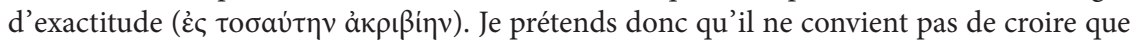
l'ancienne médecine n'existe pas et ... qu'elle ne possède pas l'exactitude dans tous les aspects, mais qu'il convient bien plutôt, pour la raison qu'elle est capable ... de s'approcher

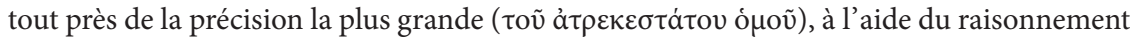
$(\lambda o \gamma \iota \sigma \mu \tilde{\omega})$, après être sortie d'une profonde ignorance, d'admirer les découvertes obtenues

\footnotetext{
14 Voir Craik $(2015: 285)$ : «a date in the final decades of the fifth century is plausible ».

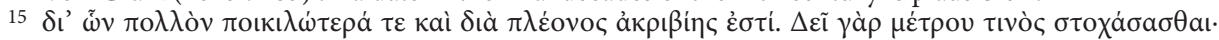

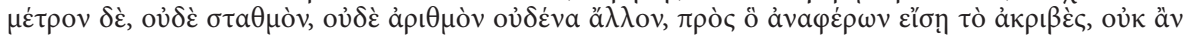

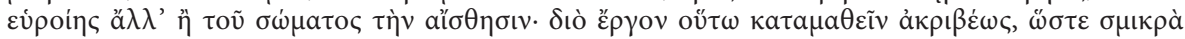

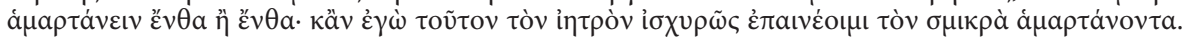

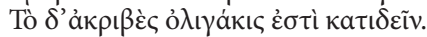


par une méthode bonne et correcte, et non sous l'effet du hasard » (VM 12, traduit par Jouanna 1990). ${ }^{16}$

Le terme atrekếs est extrêmement important dans la prose historique ionienne ; Hérodote l'utilise pour dénoter " la vérité », au sens de la certitude de son témoignage (l'adjectif alethếs n'étant usé par lui qu'exeptionellement). En général, le terme atrekếs et l'adverbe atrekéôs ${ }^{17}$ caractérisent une information ou un renseignement sans déformation et sans écart ou abérration (voir le verbe latin torqueo et les mots français dérivés torsion ; entorse etc.). Un autre adjectif / adverbe utilisé par Hérodote dans un sens comparable est asphalếs / asphaléôs, à savoir quelque chose que l'on ne peut pas ébranler ou secouer. Ce qui est substantiel ici, est la certitude du témoignage, la conviction du locuteur : si Hérodote voit quelque chose, il la qualifie comme atrekếs. En outre, on trouve dans ses Histoires d'autres termes utilisés fréquemment dans le Corpus : p. ex. saphếs qui désigne la clarté et « synopticité » du témoignage. En conséqunce, les termes atrekếs et akribếs apparaissent comme ancrés dans le discours prosaïque ionienne, bien que leur présence ne soit pas limitée à celui-ci. ${ }^{18}$

Mais les limites de la tékhnê, on les trouve aussi du coté du médecin: les maladies sont vaincues « dans la mesure où la nature des malades s'offre à l'examen et dans la mesure où la nature des investigateurs est douée pour l'investigation » (De arte 11). ${ }^{19}$ L'auteur du traité $D u$ régime utilise le raisonnement suivant pour caractériser les excellences et les limites du traitement de la part du médecin, en relation avec le malade et son régime. Selon cet auteur, le médecin qui veut traiter exactement le régime de l'homme doit connaître la nature de l'homme en général : c'est à dire ses constituants fondamentaux et ses éléments prédominants. Mais il lui faut connaître aussi la vertu de tous les aliments et boissons ainsi que la proportion des exercices à l'égard de la quantité d'aliments, de la nature du patient, de son âge, des saisons de l'année, des changements des vents etc., bref, de l'univers entier.

«Mais tout cela connu, la découverte n'est pas encore complète. Si en effet il était possible ... de trouver dans chaque cas individuel une proportion exacte des aliments et des exercises, sans excès ni défaut, on aurait trouvé alors très exactement la santé pour tout le monde. Malheureusement, si tout ce qu'on a cité plus haut a bien été découvert, ce dernier point

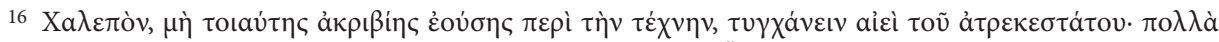

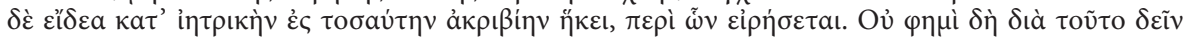

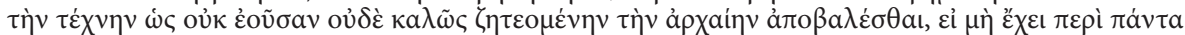

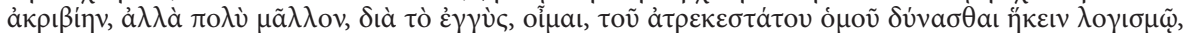

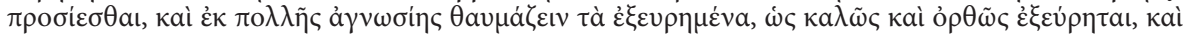

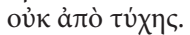

17 Sur l'usage fréquente de ces termes chez Hérodote voir Liddell, Scott, Jones (1996 : s. v. ả

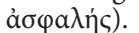

18 Pindare commence son Olymp. X par une longue exposition de l'atrékeia, suivie par le catalogue des vainqueurs de la première cérémonie olympique. Les termes utilisés dans la période préclassique pour désigner la vérité - la mensonge - la fiction forment un groupe sémantique remarquable qui a été largement étudié. Voir Luther (1935) ; Boeder (1959); Krischer (1965); Levet (1976); Cole (1983) et al.

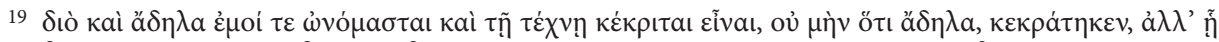

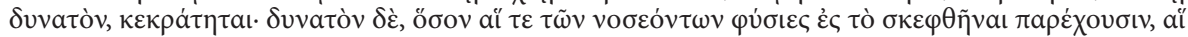

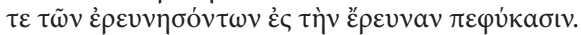


est impossible à découvrir. Si on était présent et qu'on pût observer, on connaîtrait chaque patient qui se déshabille et fait des exercices au point de le garder en santé, enlevant ici, ajoutant là, mais sans cela, il n'est pas possible de prescrire en toute exactitude aliments et exercices. J'ai dit jusqu'où pouvait aller la découverte » (Vict. 2, traduit par Joly 1967). ${ }^{20}$

Ce passage est extrêmement intéressant : on peut le rapprocher de l'argument du Politique de Platon (Pl. Plt. 294a-299e) : La tékhnê royale - ou l'art de régner - est ici comparée avec la tékhnê médicale et la timonerie.

Les hommes royaux sont ici décrits comme de vrais experts, qui s'aident de la science et de la justice et auxquels s'opposent les sociétés gouvernées par les lois, parce que « la loi ne sera jamais capable de saisir à la fois (et précisément, akribốs), ce qu'il y a de meilleur et de plus juste pour tous, de façon à édicter les prescriptions les plus utiles ». ${ }^{21}$

La cause de cette situation est « la diversité qu'il y a entre les hommes et les actes, et le fait qu'aucune chose humaine n'est, pour ainsi dire, jamais en repos, ne laisse place, dans aucun art et aucune matière, à un absolu qui vaille pour tous les cas et pour tous les temps. " 22

Et pourtant, c'est vers cela que tend la loi « comme un homme sûr de lui et ignare » ( ou les maîtres de gymnase : on peut imaginer un législateur qui - à l'instar d'un maître de gymnase - « sera capable de venir s'asseoir, à tout l'instant de la vie, auprès de chaque

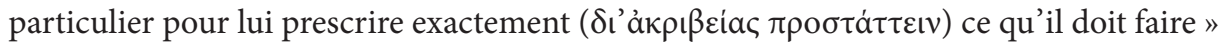
(Pl. Plt. 295a-b).

Alors, idealiter, on peut le postuler ainsi - mais, en réalité, les choses ne se déroulent jamais comme ça : il y aura les lois, lesquelles fonctionnent de manière analogue à un maître de gymnase qui, au moment de partir pour un voyage, laisse à ses élèves ou à ses clients des ordonnances générales - que l'on peut changer après un certain temps, si cela s'avère nécessaire. Dans le cas de la société humaine, c'est semblable : au lieu du souverain idéal, qui serait capable de prescrire à chaque particulier ce qu'il faut faire, il y a les lois qui se succèdent. L'analogie avec le médecin du traité $D u$ régime est, à mon avis, manifeste. Évidemment, Platon adopte ici l'argumentation de l'auteur de ce traité hippocratique. L'hypothèse qu'il l'a lu est renforcée par les ressemblances et affinités

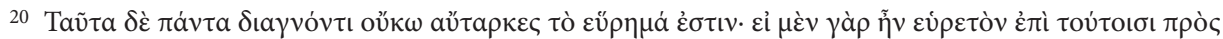

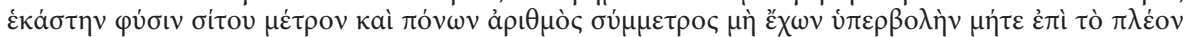

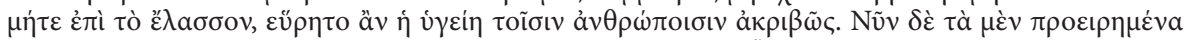

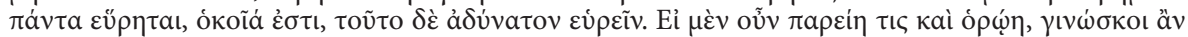

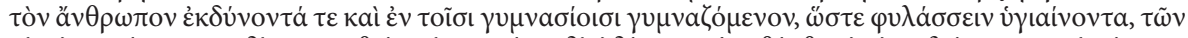

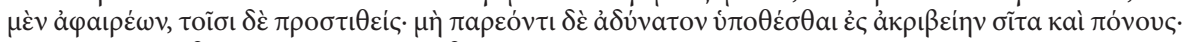

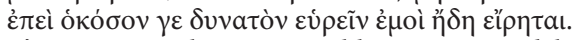

L'auteur traite le même problème aussi au début du trosième livre, pour évaluer son exploit de la

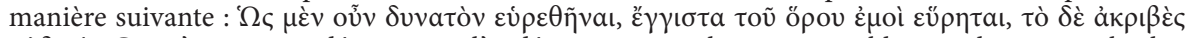
ov̉ $\delta \varepsilon v i$ « Ce qu'on pouvait découvrir, je l'ai découvert, aussi loin que possible, mais la rigueur absolue (tò dè akribès), personne ne l'a découverte » (Vict. 67, traduit par Joly 1967). Voir aussi Vict. 93.

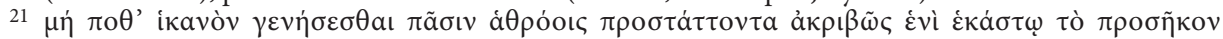

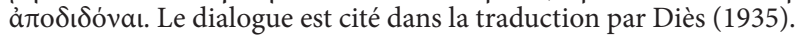

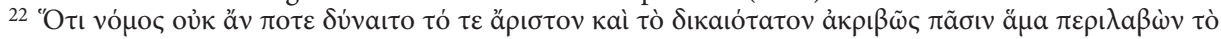

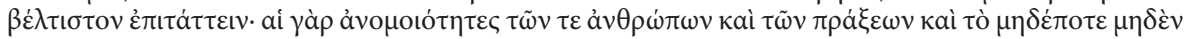

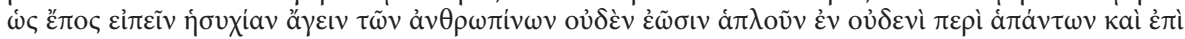

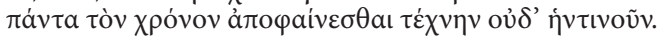


entre la théorie de la conception exposée dans la première livre de ce traité (chapitres 27-30) et le mythe de l'androgyne raconté par le poète comique Aristophane dans le Banquet de Platon. ${ }^{23}$ Selon U. Hirsch, « Platons Verständnis der ảkpißzıа Zeugnis ablegt von dem Einfluss, den das Individualprinzip der hippokratischen Medizin auf sein Denken gehabt hat. Ihr Streben, den Einzelfall in seinen Besonderheiten ernstzunehmen und ihn vom Allgemeinen her zu verstehen, macht sie zu einem hervorragenden Paradeigma der $\dot{\varepsilon} \pi \iota \tau \eta \dot{\mu} \mu$ in Platons Schriften » (Hirsch 1996 : 157).

Schiefsky comprend passage cité du Politique de manière littérale : selon Platon, commente-t-il, le professionel compétent peut atteindre l'akríbeia, à condition qu'il soit capable de considérer toutes les conditions et circonstances de chaque cas séparément. "Son image idéalisée de la compétence professionelle ne laisse aucune espace pour le manquement » (Schiefsky 2005 : 366). A la différence de Schiefsky, je propose de lire ce passage comme expérience de pensée (thought experiment, Gedankenexperiment) : l'image que nous découvrons ici - chacun a auprès de soi un législateur qui lui conseille tout ce qu'il doit faire, tout le temps et dans chaque affaire - apparaît comme une blague parfaite. A mon avis, le but de cette expérience de pensée est différent : elle vise à signaler la complexité, l'incompréhensibilité et la fluidité des choses humaines, qui sont prises dans un mouvement et un changement perpétuels.

Ajoutons qu'Aristote va encore plus loin dans ce sujet. Le Stagirite - qui était aussi fils de médecin - se plaint du manque de précision de la théorie qui traite des cas particuliers. De tels cas, argumente-t-il, ne tombent sous aucun art, sous aucune tradition profesionnelle, mais « il revient toujours à chacun, lorsqu'il agit, d'examiner ce que réclame l'occasion, tout comme on fait aussi dans la médecine et le pilotage " (Arist. Eth. Nic. 1103b-1104a). ${ }^{24}$ Ensuite, cette idée est renouée par Galien qui concevait l'art médical comme une tékhnê stochastique, caractérisée par le triptyque stokhasmós - logismós peĩra (conjecture / raisonnement / expérience). On peut voir le caractère stochastique dans son empressement à abandonner l'instrument traditionel du logismós, si celui-ci a failli. Mais ce processus remarquable sort du cadre de cet étude. ${ }^{25}$

\section{Les autres aspects spécifiques de la tékhnê médicale selon les hippocratiques}

Le médecin conçoit encore un aspect crucial de sa tékhnê - en ce qui concerne les autres artisans, tekhnĩtai, et surtout en relation avec lui-même et l'objet propre de son métier :

23 Pour savoir, si - et jusqu'à quel point - Platon connaissait les théories des traités hippocratiques, voir Bartoš (2015 : 231-241).

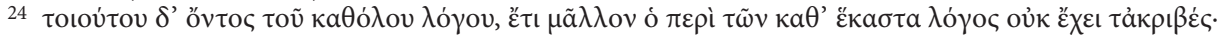

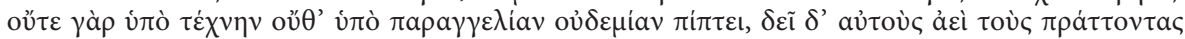

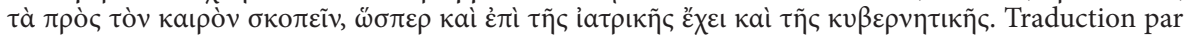
J. Y. Jolif (Gauthier, Jolif 1970).

25 Voir l'article de Boudon-Millot (2005), qui ne trouve pas d'évidence suffisante dans le Corpus pour désigner la médecine hippocratique comme stochastique (contra Ingenkamp 1983). Selon Boudon-Millot, stokházesthai se trouve à la «fuzzy » frontière entre l'art, le savoir et l'ignorance (Boudon-Millot 2005 : 90). 
«Parmi les arts, il en est certains qui sont pénibles pour leurs détenteurs mais très utiles pour leurs utilisateurs et qui apportent aux profanes un bien commun mais ne causent aux praticiens que du chagrin. A une telle catégorie d'arts appartient précisément celui que les Grecs appellent médecine. En effet le médecin voit des spectacles effrayants, touche des choses répugnantes, et à l'occasion des malheurs d'autrui récolte pour lui-même des chagrins. Les malades au contraire échappent, grâce à l'art, aux maux les plus grands, maladies, affliction, souffrances, mort ; car c'est à tout cela que s'oppose la médecine » (Flat. 1, traduit par Jouanna 1988). ${ }^{26}$

Cette caractéristique était très populaire et célèbre, encore plus célèbre que l'apho-

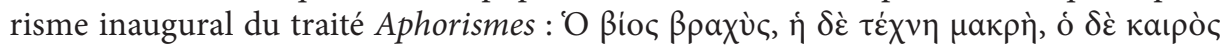

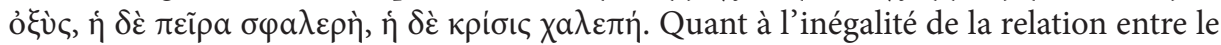
médecin et le malade, elle était commentée dans le Corpus maintes fois :

«Ce ne sont pas de petits rapports que ceux du médecin avec les malades: les malades se soumettent au médecin, et lui, à toute heure, est en contact avec des femmes, avec de jeunes filles, avec des objets précieux; il faut, à l'égard de tout cela, garder les mains pures » (Med. 1). ${ }^{27}$

On trouve des appels et incitations à respecter les principes de la justice, principe de l'utilité, de ne pas porter dommage et de ne pas abuser de sa position supérieure, dans les traités déontologiques de Corpus Hippocraticum assez souvent, et aussi bien dans le Serment d'Hippocrate où on lit : "Je m'abstiendrai de tout mal et de toute injustice. ... Dans quelque maison que j'entre, j'y entrerai pour l'utilité des malades, me préservant de toute méfait volontaire et corrupteur, et surtout de la séduction des femmes et des garçons, libres ou esclaves ». ${ }^{28}$ De plus, le médecin doît être philánthrôpos - c'est à dire philanthrope ou, simplement, humain - cela appartient à son métier même : "Où il y a l'amour pour

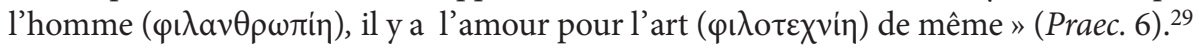

Les traités déontologiques du Corpus comprennent aussi les instructions pratiques pour le médecin, en égard à l'étiquette médicale : comment le médecin doit s'habiller, se conduire, parler, rire, couper ses ongles etc. Ces prescriptions doivent être interprétées à la lueur de la pratique médicale en Grèce antique : l'éducation médicale n'étant pas obligatoire, chacun pouvait se déclarer médecin et pratiquer ce métier. Parmi la bande de charlatans, rébouteux, guérisseurs trompeurs et magiciens, le médecin hippocratique devait convaincre le malade de sa compétence et de l'efficacité de son traitement. ${ }^{30}$

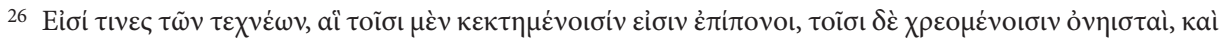

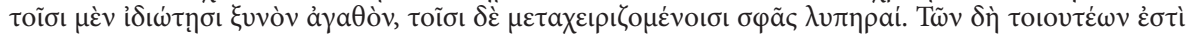

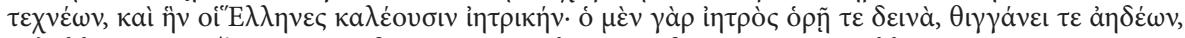

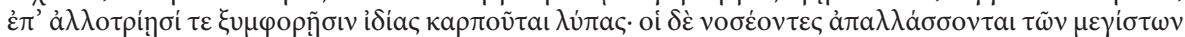

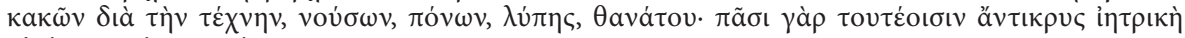

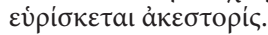

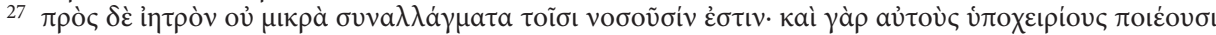

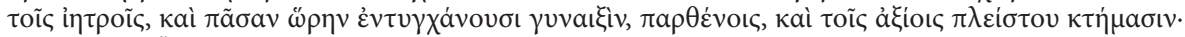

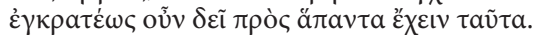

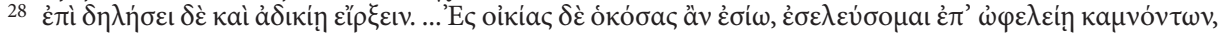

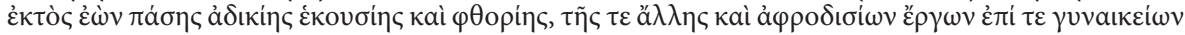

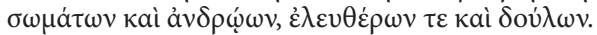

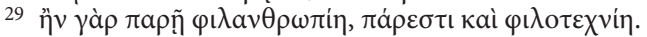

30 Pour des renseignements générals sur ce sujet voir Nutton (2004). 
Le régime, díaita, constituait une part importante de ce traitement, comme nous l'avons déjà évoqué brièvement en rapport avec le passage du dialogue Politique de Platon. L'invention de díaita en général a été considérée comme la découverte majeure des médecins hippocratiques. C'est dans ce sens qu'argumente l'auteur de L'ancienne médecine, quand il explique la découverte originaire des aliments spécifiques pour les hommes (en contraste avec les aliments crus et bruts consommés par les animaux). L'invention des aliments spécifiques pour les malades est présentée ici comme la continuation naturelle de cette invention première (VM 3-5), toutes les deux résultant de la même méthode

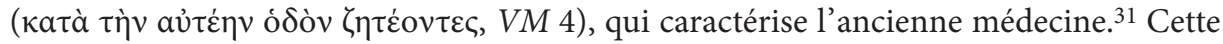
exposition détaillée documente bien un autre trait essentiel de la tékhnê hippocratique, c'est à dire l'intérêt de la discipline à son histoire propre.

Quant au régime, il comprenait dans son ensemble des composantes nombreuses : les aliments et les boissons (qui sont discutées très précisément), les exercices, le travail, les promenades, les bains, le sommeil et les songes, mais aussi la fréquence des activités sexuelles. Le malade, bien entendu, doit accepter beaucoup de restrictions, en ce qui concerne les aliments et les autres activités ; mais - et c'est crucial - le médecin hippocratique adresse ses prescriptions aussi aux hommes sains (nous les lisons p. ex. dans les traités $D u$ régime salutaire et $D u$ régime). A cet égard, l'auteur $D u$ régime distingue deux sortes des hommes: d'un côté, il'y a la grande masse des gens qui « mangent et boivent au petit bonheur » et qui sont obligés de travailler (Vict. 68; toutes les traductions du traité par Joly 1967), sans pouvoir négliger tout le reste pour s'occuper de leur santé. De l'autre côté, il'y a ceux qui ont les moyens et qui ont reconnu « que les richesses ni rien d'autre n’ont d'utilité sans la santé » (Vict. 69). Quoique l'auteur du traité présente les conseils diétetiques aussi pour la première sorte des gens, il se concentre à la seconde : pour elle, il a découvert « un régime qui s’avance le plus près possible de la vérité absolue » ( $\pi \rho$ ò

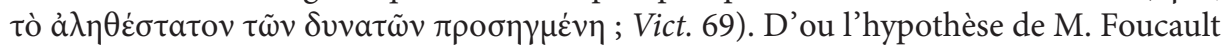
selon laquelle le traité représentrait un manuel qui n'était pas adressé aux médecins ou aux maîtres de gymnase mais aux laïques - et particulièrement à cette deuxième groupe des gens « avec moyens » mentionée auparavant.

Tout cela signifie que l'exigence diététique est de la nature éthique : díaita charge les hommes d'un régime de restrictions, de limitations, d'abstinence de telle ou telle sorte, bref : de discipline (on peut déjà observer ce trait sémantique dans le verbe apperenté diaitỗ, qui peut signifier gouverner - diriger - réguler - modérer). Michel Foucault était fasciné par ce trait spécifique de la médecine hippocratique ; il écrit, dans le deuxième tome de l'Histoire de la sexualité, que le régime est pour l'homme une catégorie fondamentale : il permet d'inscrire dans sa conduite « un mode de problématisation du comportement ", c'est à dire une auto-régulation qui l'empêche de s'exposer à ses désirs et plaisirs - qui sont, naturellement, sans limites, et donc pernicieux. En somme, «le régime est tout un art de vivre ", une " technique », ou même une " esthétique de l'existence ", pas seulement un ensemble de précautions destinées à éviter les maladies ou à achever de les guérir (Foucault $1984: 115-123) .{ }^{32}$

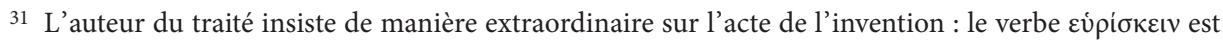

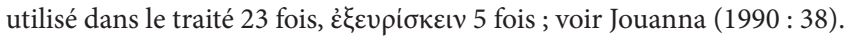

32 Bartoš argumente que « matters of morality and value judgement are clearly distinguished on several occasions from matters of health in the Hippocratic text » (i.e. in Du régime; Bartoš 2015 : 218) et cette 
De là vient aussi la querelle entre la médecine et la philosophie, qui - surtout dans les dialogues de Platon - prétend avoir la seule autorité éthique, parce qu'elle prend soin de l'âme de l'homme, et pas seulement de son corps. ${ }^{33}$

On peut conclure de la manière suivante : au point de vue éthique, l'art médical impose les exigences non seulement à la personne du médecin, mais aussi à la personne du malade. Mais, si l'attitude du malade dépend de sa volonté, le médecin n'a aucun choix. Il est dans la nature même de l'art médical que son tekhnitês, le médecin, doive se montrer plus humain et, au plan moral, supérieur au reste de la société. On le voit clairement dans une phrase, qui, dans la composition circulaire du Serment d'Hippocrate, tient lieu de centre et de point focal ${ }^{34}$ : « Je passerai ma vie et j'exercerai mon art dans la pureté et la piété ».35

Il semble que l'art lui même, dont le médecin est le serviteur, serait apotheosé et déifié ici - malgré toutes ses limitations mentionnées auparavant.

\section{RÉFÉRENCES}

Allen, Th. W., 1912. Homeri opera. Tomus V. Hymnos Cyclum fragmenta Margiten Batrachomyomachiam Vitas continens. Recognovit brevique adnotatione critica instruxit Th. W. Allen. Oxford : Clarendon Press.

Bartoš, H., 2015. Philosophy and Dietetics in the Hippocratic On Regimen. Leiden / Boston : Brill.

Beekes, R., 2010. Etymological Dictionary of Greek. I-II. Leiden / Boston : Brill.

Boeder, H., 1959. «Der frühgriechische Wortgebrauch von Logos und Aletheia ». Archiv für Begriffsgeschichte 4, 82-112.

Boschung, U., 2003. « Der hippokratische Eid - Überlieferung, Wirkungsgeschichte und medizinhistorische Interpretation ». In: B. Ausfeld-Hafter (ed.), Der hippokratische Eid und die heutige Medizin. Bern / Berlin / Wien : Lang, 9-26.

Boudon-Millot, V., 2005. «Art, Science and Conjecture, from Hippocrates to Plato and Aristotle ». In: P. J. Van der Eijk (ed.), Hippocrates in Context. Leiden / Boston : Brill, 87-99.

Brătescu, G., 1983. « Le problème de la mesure dans la Collection hippocratique ». In: Lasserre, Mudry (1983: 137-144).

CAF $=$ Comicorum Atticorum fragmenta. Volumen I.-Volumen III. Edidit Th. Kock. Lipsiae: in aedibus B. G. Teubneri, $1880-1888$.

Cole, T., 1983. « Archaic Truth ». Quaderni Urbinati di Cultura Classica 13, 7-28.

Craik, E. M., 2015. The Hippocratic Corpus. Content and Context. London / New York : Routledge.

Delkeskamp-Hayes, C., Gardell Cutter, M. A. (ed.), 1993. Science, Technology, and the Art of Medicine. European-American Dialogues. Dordrecht / Boston / London : Kluwer Academic Publishers.

Diès, A., 1935. Platon, Le politique. Texte établi et traduit par A. Diès. Paris : Les Belles Lettres.

proposition est correcte, mais cela ne signifie pas que l'exigence basée sur l'individuel sain, quant au respect d'un certain régime, n’a pas une nature éminemment éthique. Voir déjà Schumacher : díaita « bezeichnet ganz allgemein die Lebensweise eines Menschen, dann aber auch die Anordnung dazu, und zwar in ethischer und physischer Hinsicht» (Schumacher 1963 : 58).

33 Il me faut préciser que dans les traités hippocratiques on rencontre l'âme assez souvent, mais elle se comporte toujours de manière similaire au corps. La distinction essentielle que possède l'âme dans les dialogues de Platon est absente des traités du Corpus Hippocraticum, voir Bartoš (2015 : 165-229).

34 Pour la position centrale de cette obligation dans le Serment voir Boschung, qui la qualifie comme « das zentrale Versprechen » (Boschung 2003 : 10); dans la manière semblable argumentent von Staden $(1996: 417)$ et Lichtenthaeler (1984: 153).

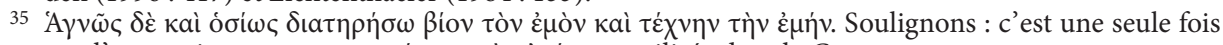

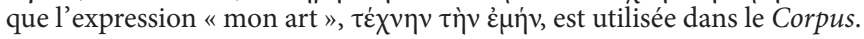


Foucault, M., 1984. Histoire de la sexualité 2. L'usage des plaisirs. Paris : Gallimard.

Gadamer, H.-G., 1996. The Enigma of Health. Translated by J. Gaiger and N. Walker. Stanford : Stanford University Press.

Gauthier, R. A., Jolif, J.-Y., 1970. Aristote, L'éthique à Nicomaque. Tome I. Deuxième partie. Traduction. Deuxième édition. Introduction, traduction et commentaire par R. A. Gauthier et J. Y. Jolif. Louvain : Publications Universitaires / Paris : Beatrice-Nauwelaerts.

Heinimann, F., 1961. «Eine vorplatonische Theorie der techne ». Museum Helveticum 18, 105-130.

Hirsch, U., 1996. « Akribeia - Platons Verständnis der episteme und die hippokratische Medizin ». In: Wittern, Pellegrin (1996: 149-158).

Ingenkamp, H.-G., 1983. « Das stochasasthai des Arztes (VM 9) ». In: Lasserre, Mudry (1983 : 257-262). Joly, R., 1967. Hippocrate, Du régime. Texte établi et traduit par R. Joly. Paris : Les Belles Lettres.

Jouanna, J., 1988. Hippocrate, Des vents. De l'art. Texte établi et traduit par J. Jouanna. Paris : Les Belles Lettres.

Jouanna, J., 1990. Hippocrate, L'ancienne médecine. Texte établi et traduit par J. Jouanna. Paris : Les Belles Lettres.

Krischer, T., 1965. «Etymos und alethes ». Philologus 109, 161-174.

Kube, J., 1969. Techne und arete. Sophistisches und platonisches Tugendwissen. Berlin : Walter de Gruyter.

Kurz, D., 1970. Akribeia: Das Ideal der Exaktheit bei den Griechen bis Aristoteles. Göppingen : Verlag Alfred Kummerle.

Lasserre, F., Mudry, P. (ed.), 1983. Formes de pensée dans la Collection hippocratique. Genève : Droz.

Levet, J. P., 1976. Le vrai et le faux dans la pensée grecque archaïque. Paris : Les Belles Lettres.

Lichtenthaeler, C., 1984. Der Eid des Hippokrates. Ursprung und Bedeutung. Köln : Deutscher Ärzteverlag.

Liddell, H. G., Scott, R., Jones, H. S., 1996. A Greek-English Lexicon. Ninth ed. With a revised supplement 1996. Oxford: Clarendon Press.

Littré, É., 1839-1861. Euvres complètes d'Hippocrate. I-X. Traduction nouvelle avec le texte grec en regard, collationné sur les manuscrits ... par E. Littré. Paris : Baillière.

Luther, W., 1935. „Wahrheit“ und „Lüge“ im ältesten Griechentum. Borna / Leipzig : R. Noske.

Nussbaum, M. C., 1989. The Fragility of Goodness. Luck and Ethics in Greek Tragedy and Philosophy. Cambridge : Cambridge University Press.

Nutton, V., 2004. Ancient Medicine. London / New York: Routledge.

Schiefsky, M. J., 2005. Hippocrates, On Ancient Medicine. Translated with introduction and commentary by M. J. Schiefsky. Leiden / Boston : Brill.

Schumacher, J., 1963. Antike Medizin. Die naturphilosophischen Grundlagen der Medizin in der griechischen Antike. Zweite verbesserte Aufl. Berlin : Walter de Gruyter.

Staden, H. von, 1996. "'In a Pure and Holy Way': Personal and Professional Conduct in the Hippocratic Oath? ». Journal for the History of Medicine and Allied Sciences 51, 404-437.

Thivel, A., 1985. « Diagnostic et pronostic à l'époque d'Hippocrate et à la nôtre ». Gesnerus 42, 479-497.

Villard, L., 1996. "Les médecins hippocratiques face au hasard, ou le recours alterné à l'archéologie et à l'étymologie ». In: Wittern, Pellegrin (1996:395-412).

Wittern, R., Pellegrin, P. (ed.), 1996. Hippokratische Medizin und antike Philosophie. Hildesheim : Olms-Weidmann.

\section{TECHNICITA HIPPOKRATOVSKÉHO LÉKAŘSTVÍ A JEJÍ MEZE}

Studie se věnuje hippokratovskému konceptu techné, který tvořil součást širších debat o techné $\mathrm{v}$ Recku 5. stol. př. Kr. Koncept techné je zakotven v konceptu fysis (a je v opozici vůči pojmu tyché); o jeho závažnosti svědčí fakt, že před časem označil H.-G. Gadamer objev techné a jeho aplikaci na lékařství za první rozhodující krok směřující k ustavení západní civilizace. Nejprve jsou představeny základní rysy techné iétriké, formulované ve spisech Corpus Hippocraticum: lékařství si je vědomo svého cíle i své metody, jejiž povahu opakovaně reflektuje. K této reflexi patří vědomí určitých mezí na straně lékařství, a to zejména nedostatku akribeia, přesnosti, nebot lékař se vztahuje k objektům jiného rázu než ostatní 
umění: k jedinečnému ustrojení každého pacienta. Dalším specifikem lékařské techné je nerovný vztah mezi odborníkem-lékařem a objektem jeho umění, tedy pacientem; tato skutečnost je v rámci Corpus Hippocraticum vícekrát komentována. V závěru je poukázáno na etický rozměr dietetických preskripcí i lékařské profese jako takové, který - i přes veškerá omezení uvedená výše - vede téměř ke zbožnění lékařského umění i života.

Sylva Fischerová

Université Charles, Prague

sylva.fischerova@ff.cuni.cz 